\title{
MAXIMAL SEPARABLE SUBFIELDS
}

\author{
BONNIE PAGE DANNER
}

\begin{abstract}
If $L / K$ is a finitely generated separable field extension of characteristic $p \neq 0$ and $M$ is an intermediate field such that $L / M$ is inseparable, it is proved there exist subfields $S$ of $M$ maximal with respect to the property that $L / S$ is separable. These maximal separable subfields, denoted $S$-subfields for $L / M$, are characterized in two ways.

(1) Let $L / S$ be a separable field extension. Then $S$ is a $S$-subfield for $L / M$ if and only if $S\left(L^{p}\right) \supseteq M$ and $S$ is algebraically closed in $M$.

(2) If $L / S$ is separable, $S$ is a $S$-subfield for $L / M$ if and only if the inseparability of $L / M$ is equal to the transcendence degree of $M / S$.

A $S$-subfield for $L / M$ is constructed using a maximal subset of a relative $p$-basis for $M / K$ which remains $p$-independent in $L$. It is proved that there is a unique $S$-subfield for $L / M$ if and only if $S / K$ is algebraic for some $S$.
\end{abstract}

Throughout this paper $L / K$ will be a finitely generated separable field extension of characteristic $p \neq 0$, and $M$ will be an intermediate field such that $L / M$ is inseparable. In this discussion we investigate subfields $S$ of $M / K$ which are maximal with respect to the property that $L$ is separable over $S$. These maximal separable subfields are denoted $S$-subfields for $L / M$.

First we prove the existence of $S$-subfields for $L / M$ and then illustrate one possible subfield by construction, using a subset of a relative $p$-basis for $M / K$ which is maximal with respect to the property that the subset remains $p$-independent in $L$. Characterizations of $S$-subfields for $L / M$ are given by two theorems. Theorem 7 states if $L / S$ is separable, $S$ is a $S$-subfield for $L / M$ if and only if $S\left(L^{p}\right) \supseteq M$ and $S$ is algebraically closed in $M$. We note that $M$ will be a regular extension of any $S$-subfield for $L / M$. Theorem 8 states if $L / S$ is regular, $S$ is a $S$-subfield for $L / M$ if and only if the inseparability of $L / M$ is equal to the transcendence degree of $M / S$. From this result we obtain that all $S$-subfields for $L / M$ have the same transcendence degree over $K$. We then determine properties of $S$-subfields for $L / M$ in relation to some of the intermediate fields of $L / K$. Beginning with $S / K$, a finitely generated separable extension, and $M$, a regular extension of $S$, we construct a $L$ such that $S$ is a $S$-subfield for $L / M$. Finally, we show in Theorem 14 that there will exist a unique $S$-subfield for $L / M$ if and only if $S / K$ is algebraic for some $S$, where $S$ is a $S$-subfield for $L / M$. A field $L$ is said to be separable over a subfield $K$ if and only if $L^{p}$ (the field of $p$ th powers of $L)$ and $K$ are linearly disjoint over $K^{p}$. The inseparability of $L / M$, denoted

Received by the editors April 11, 1977.

AMS (MOS) subject classifications (1970). Primary 12F15.

Key words and phrases. Separable and inseparable field extensions, $p$-bases.

(C) American Mathematical Society 1978 
insep $L / M$, is equal to the number of elements in a relative $p$-basis for $L / M$ minus the number of elements in a transcendency basis for $L / M$.

Definition 1. If $L / K$ is separable and finitely generated and if $L$ is inseparable over an intermediate field $M$, then a subfield $S$ of $M$ containing $K$ is a $S$-subfield for $L / M$ if and only if $S$ is a maximal subfield of $M$ with respect to the property that $L / S$ is separable.

The following known result is used to prove the existence of $S$-subfields for $L / M$.

LEMMA 2. If $L / K$ is a finitely generated field extension, then any intermediate field is finitely generated over $K$.

THEOREM 3. If $L / K$ is finitely generated and separable, and $L$ is inseparable over an intermediate field $M$, then there exist $S$-subfields for $L / M$.

Proof. Let $\mathcal{S}$ be the collection $S_{\alpha}$ of subfields of $M / K$ such that $L$ is separable over each $S_{\alpha}$. Let set inclusion be a partial ordering on $\delta$. Since $L / K$ is separable, $K \in \mathcal{S}$ and $\delta \neq \varnothing$. Let $\mathcal{C}$ be a chain in $\delta$ and let $E=\cup_{S_{\alpha} \in \mathcal{C}} S_{\alpha}$, which is clearly an upper bound of $\mathcal{C}$. Since $\mathcal{C}$ is a totally ordered subcollection of $S, E$ must be a subfield of $M / K$. By Lemma $2, E$ is finitely generated over $K$. Let $E=K\left(\beta_{1}, \beta_{2}, \ldots, \beta_{r}\right)$. By the definition of $E$, $\beta_{1}, \beta_{2}, \ldots, \beta_{r} \in S_{\alpha}$ for some $\alpha$. Hence, $K\left(\beta_{1}, \beta_{2}, \ldots, \beta_{r}\right)=S_{\alpha}$ and $L / E$ is separable. This implies $E \in \mathcal{S}$. By Zorn's Lemma there exists a maximal element of $\mathcal{S}$. We conclude there exist $S$-subfields for $L / M$.

In general there does not exist a unique $S$-subfield for $L / M$. We show in Theorem 14 precisely when there will be a unique maximal subfield. A $S$-subfield for $L / M$ can be constructed using a subset of a relative $p$-basis for $M / K$ which remains $p$-independent in $L$. Let $B$ be a maximal subset of a relative $p$-basis for $M / K$ which remains $p$-independent in $L$. Then $L / K(B)$ is separable [5, p. 378].

Proposition 4. Let $L / S$ be separable where $L \supset M \supset S \supset K(B) \supset K$. If $B$ is a maximal subset of a relative $p$-basis for $M / K$ which remains $p$-independent in $L$, then $S$ must be an algebraic extension of $K(B)$.

Proof. Suppose $S$ is a transcendental extension of $K(B)$. We noted above that $L / K(B)$ is separable; hence, $S / K(B)$ is separable and has a separating transcendency basis [5, Theorem 3, p.373]. Let $T$ be a separating transcendency basis for $S$ over $K(B)$. The basis $T \neq \varnothing$ since $S / K(B)$ is transcendental. Let $B \cup T$ be a relative $p$-basis for $S / K$. Since $L / S$ is separable, $B \cup T$ remains relatively $p$-independent in $L / K[5$, p. 378]. This implies $B \cup T$ is relatively $p$-independent in $M / K$ and contradicts the hypothesis that $B$ is a maximal subset of a relative $p$-basis for $M / K$ which remains $p$-independent in $L$. We conclude $S$ is an algebraic extension of $K(B)$.

Therefore, any extension $S$ of $K(B)$ contained in $M$ with the property that $L / S$ is separable must be algebraic. A $S$-subfield for $L / M$ will be the largest 
algebraic extension of $K(B)$ in $M$. Hence, the algebraic closure of $K(B)$ in $M$ is a $S$-subfield for $L / M$.

Proposition 5. The field extension $M / S$ is not algebraic.

Proof. Since $L / S$ is separable, $M / S$ is separable. If $M / S$ is algebraic, then $L / M$ is separable [1, p. 8]. This contradicts the assumption that $L / M$ is inseparable. Hence, $M / S$ is not algebraic.

Proposition 6. The field $S$ is algebraically closed in $M$.

Proof. Let $s \in M$ and $s$ be algebraic over $S$. Then $L / S(s)$ must be separable since $L / S$ is separable and $S(s) / S$ is algebraic. Since $S$ is a $S$-subfield for $L / M, S$ is a maximal subfield of $M$ with respect to the separability of $L / S$; hence $s$ must belong to $S$, and we conclude that $S$ is algebraically closed in $M$.

The next two theorems are useful characterizations of $S$.

THEOREM 7. If $L / S$ is separable, $S$ is a $S$-subfield for $L / M$ if and only if $S\left(L^{p}\right) \supseteq M$ and $S$ is algebraically closed in $M$.

Proof. Suppose $S$ is a $S$-subfield for $L / M$. We have shown that $S$ is algebraically closed in $M$. Let $\theta \in M$ but $\theta \notin S$. The element $\theta$ must be transcendental over $S$; hence, $\{\theta\}$ is a relative $p$-basis for $S(\theta) / S$. Since $S$ is maximal in $M, L / S(\theta)$ cannot be separable. Therefore, $\theta$ is not relatively $p$-independent in $L / S$. Let $B$ be a $p$-basis for $S$. Then $B \cup\{\theta\}$ is a $p$-basis for $S(\theta)$. Since $B$ remains $p$-independent in $L$ by the separability of $L / S$ and since $B \cup\{\theta\}$ does not remain $p$-independent in $L, \theta \in L^{p}(B) \subseteq S\left(L^{p}\right)$. Since $\theta$ was arbitrary, $M \subseteq S\left(L^{p}\right)$. Conversely, suppose $S$ is algebraically closed in $M$ and $M \subseteq S\left(L^{p}\right)$. Consider an extension $S^{\prime}$ of $S$ contained in $M$. Let $B^{\prime}$ be a relative $p$-basis for $S^{\prime} / S$. The basis $B^{\prime} \neq \varnothing$ since $S^{\prime} / S$ must be transcendental. From the hypothesis and since $B^{\prime} \subseteq M, S\left(L^{p}\right)=S\left(L^{p}, B^{\prime}\right)$ which implies that $B^{\prime}$ does not remain relatively $p$-independent in $L$, and $L / S^{\prime}$ cannot be separable. Hence, $S$ is a maximal subfield of $M$ such that $L / S$ is separable.

Let $p_{L / M}, p_{L / S}$, and $p_{M / S}$ represent relative $p$-bases for $L / M, L / S$, and $M / S$, respectively.

TheORem 8. Let $L / S$ be separable. Then $S$ is a $S$-subfield for $L / M$ if and only if $\operatorname{tr} \mathrm{d} M / S=$ insep $L / M$ and $S$ is algebraically closed in $M$.

Proof. Suppose $S$ is a $S$-subfield for $L / M$. Since $p_{L / M}$ and $p_{L / S}$ are relative $p$-bases for $L / M$ and $L / S$ respectively, by definition $L=$ $M\left(L^{p}, p_{L / M}\right)$ and $L=S\left(L^{p}, p_{L / S}\right)$. By Theorem $7, M \subseteq S\left(L^{p}\right)$, therefore $L=S\left(L^{p}, p_{L / M}\right)$. Hence, a relative $p$-basis for $L / M$ will be a relative $p$-basis for $L / S$ and $\left|p_{L / M}\right|=\left|p_{L / S}\right|$.

We note that

$$
\operatorname{tr} \mathrm{d} L / S=\operatorname{tr} \mathrm{d} L / M+\operatorname{tr} \mathrm{d} M / S
$$


By the separability of $L / S$ there exists a separating transcendency basis for $L / S$ [5, Theorem 3, p. 373] and $\operatorname{tr} \mathrm{d} L / S=\left|p_{L / S}\right|$ [5, Lemma 3, p. 382]. From the definition of inseparability we obtain the equality

$$
\operatorname{tr} \mathrm{d} L / M+\operatorname{insep} L / M=\left|p_{L / M}\right|
$$

therefore,

$$
\operatorname{tr} \mathrm{d} L / M+\operatorname{insep} L / M=\left|p_{L / S}\right|
$$

Equating (8.a) and (8.b), we obtain

$$
\operatorname{tr} \mathrm{d} L / M+\operatorname{tr} \mathrm{d} M / S=\operatorname{tr} \mathrm{d} L / M+\operatorname{insep} L / M
$$

Hence,

$$
\operatorname{tr} \mathrm{d} M / S=\operatorname{insep} L / M \text {. }
$$

By Proposition 6, $S$ is algebraically closed in $M$. Conversely, suppose $\operatorname{tr} \mathrm{d} L / S=$ insep $L / M$ and $S$ is algebraically closed in $M$. If $S$ is not a maximal subfield of $M$ such that $L / S$ is separable, then $S$ is contained in some $S^{\prime}$ which is a $S$-subfield for $L / M$. By the first part of this theorem $\operatorname{tr} \mathrm{d} M / S^{\prime}=$ insep $L / M$. By hypothesis $\operatorname{tr} \mathrm{d} M / S^{\prime}=\operatorname{tr} \mathrm{d} M / S$; therefore, $S^{\prime}$ must be algebraic over $S$. Since $S$ is algebraically closed in $M, S=S^{\prime}$, and $S$ is a $S$-subfield for $L / M$.

COROllaRY 9. All $S$-subfields for $L / M$ have the same transendence degree over $K$.

Proof. This follows easily from the fact that insep $L / M=\operatorname{tr} \mathrm{d} M / S$ for any $S$-subfield for $L / M$.

We note that a $S$-subfield for $L / M$ is algebraically closed in $M$, and $M / S$ is a separable extension. Hence, $M$ is a regular extension of each $S$-subfield for $L / M$.

Assume that $L / M$ is an algebraic extension and $L$ is inseparable over $M$. There exists a unique intermediate field $M^{\prime}$ such that $M^{\prime} / M$ is separable and $L / M^{\prime}$ is purely inseparable [2, pp. 46-47].

THEOREM 10. If $L / M$ is algebraic and $M^{\prime}$ is the intermediate field such that $L / M^{\prime}$ is purely inseparable and $M^{\prime} / M$ is separable, then the algebraic closure in $M^{\prime}$ of a $S$-subfield for $L / M$ will be a $S$-subfield for $L / M^{\prime}$. Moreover, if $S^{\prime}$ is a $S$-subfield for $L / M^{\prime}$ and $S^{\prime} /\left(S^{\prime} \cap M\right)$ is algebraic, then $S^{\prime} \cap M$ is a $S$-subfield for $L / M$.

ProOF. 


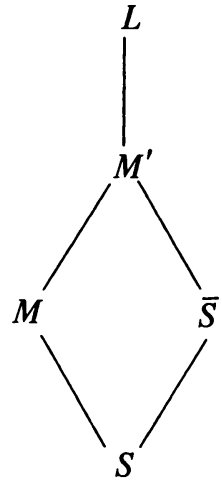

FIGURE (i)

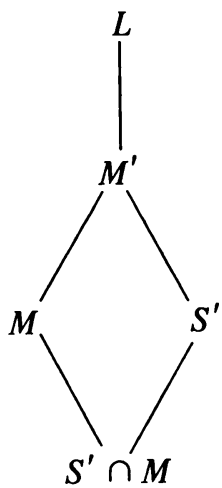

FIGURE (ii)

(i) Suppose $\bar{S}$ is the algebraic closure in $M^{\prime}$ of $S$, where $S$ is a $S$-subfield for $L / M$. Since $M^{\prime} / M$ is algebraic and $\bar{S}$ is algebraically closed in $M^{\prime}$ with $S$ algebraically closed in $M, \operatorname{tr} \mathrm{d} M^{\prime} / \bar{S}=\operatorname{tr} \mathrm{d} M / S$. By Theorem $8, \operatorname{tr} \mathrm{d} M / S$ = insep $L / M$ and since $M^{\prime} / M$ is separable, insep $L / M=\operatorname{insep} L / M^{\prime}[3$, p. 112]. Hence, $\operatorname{tr} \mathrm{d} M^{\prime} / \bar{S}=$ insep $L / M^{\prime}$. Since $L / S$ is separable and $\bar{S} / S$ is algebraic, $L / \bar{S}$ is separable. By Theorem $8, \bar{S}$ is a $S$-subfield for $L / M$.

(ii) Suppose $S^{\prime}$ is a $S$-subfield for $L / M^{\prime}$ and $S^{\prime}$ is algebraic over $S^{\prime} \cap M$. To show $S^{\prime} \cap M$ is algebraically closed in $M$, let $\alpha \in M$ and $\alpha$ be an algebraic element over $S^{\prime} \cap M$. Since $\alpha \in M^{\prime}$ and $S^{\prime}$ is algebraically closed in $M^{\prime}, \alpha \in S^{\prime}$. Hence, $\alpha \in S^{\prime} \cap M$. Since $M^{\prime} / M$ is separable, insep $L / M=$ insep $L / M^{\prime}$. The extension $M^{\prime} / M$ is algebraic and $S^{\prime} /\left(S^{\prime} \cap M\right)$ is algebraic; hence, $\operatorname{tr} \mathrm{d} M^{\prime} / S^{\prime}=\operatorname{tr} \mathrm{d} M /\left(S^{\prime} \cap M\right)$. We see that $\operatorname{tr} \mathrm{d} M /\left(S^{\prime}\right.$ $\cap M)=\operatorname{insep} L / M^{\prime}=\operatorname{insep} L / M$ since $S^{\prime}$ is a $S$-subfield for $L / M^{\prime}$ [Theorem 8]. Finally, we must show that $L /\left(S^{\prime} \cap M\right)$ is separable. Since $M^{\prime} / S^{\prime}$ is separable and $M^{\prime} / M$ is separable, $M^{\prime}$ is separable over $S^{\prime} \cap M$. The field $S^{\prime}$ is a subfield of $M^{\prime}$; hence, $S^{\prime}$ is separable over $S^{\prime} \cap M$. Then since $L / S^{\prime}$ is separable and $S^{\prime} /\left(S^{\prime} \cap M\right)$ is separable, it follows that $L /\left(S^{\prime} \cap M\right)$ is separable. We conclude that $S^{\prime} \cap M$ is a $S$-subfield for $L / M$.

More generally, let $L / M$ be an inseparable extension and $S$ be a $S$-subfield for $L / M$.

Proposition 11. If $E$ is an intermediate field of $M / S$ where $S$ is a $S$-subfield for $L / M$, then $S$ is a $S$-subfield for $L / E$.

Proof. This follows easily from Theorem 7.

Proposition 11 implies that a relative $p$-basis for $L / M$ will be a relative $p$-basis for $L / E$ and will also be a relative $p$-basis for $L / S$ [Theorem 7].

COROLlaRy 12. If $E$ is an intermediate field of $M / S$ where $S$ is a $S$-subfield for $L / M$ and $M / E$ is algebraic, then insep $L / M=\operatorname{insep~} L / E$. 
Proof. Since $S$ is a $S$-subfield for $L / E$, insep $L / E=\operatorname{tr} \mathrm{d} E / S=$ $\operatorname{tr} \mathrm{d} M / S=$ insep $L / M$ by Theorem 8 .

Suppose $S$ is a separable extension of $K$ and $M$ is a regular extension of $S$. A field $L$ can be constructed such that $L / M$ is inseparable and $S$ is a $S$-subfield for $L / M$.

THEOREM 13. If $S / K$ is a separable extension and $M / S$ is regular, there exists an inseparable extension $L / M$ such that $S\left(L^{p}\right) \supseteq M$ and $L / S$ is separable.

Proof. The field $M$ is finitely generated over $S$. Let $M=S\left(t_{1}, t_{2}, \ldots, t_{s}\right.$, $\alpha)$ where $\left\{t_{1}, t_{2}, \ldots, t_{s}\right\}$ is a separating transcendency basis for $M / S$. Let $L=M\left(t_{1}^{1 / p}, t_{2}^{1 / p}, \ldots, t_{s}^{1 / p}\right)$. Clearly, $S\left(L^{p}\right) \supseteq M$. By hypothesis $M / S$ is separable and

$$
M\left(t_{1}^{1 / p}, t_{2}^{1 / p}, \ldots, t_{s}^{1 / p}\right)=S\left(t_{1}^{1 / p}, t_{2}^{1 / p}, \ldots, t_{s}^{1 / p}, \alpha\right) .
$$

Since $t_{1}, t_{2}, \ldots, t_{s}$ are algebraically independent over $S$, their $p$ th roots are also algebraically independent over $S$. The element $\alpha$ is separable over $S\left(t_{1}\right.$, $t_{2}, \ldots, t_{s}$ ) by construction. Hence $L / S$ is separable.

Clearly, the $L$ constructed in Theorem 13 is a minimal extension of $M / S$ such that $S$ is a $S$-subfield for $L / M$.

THEOREM 14. There will exist a unique $S$-subfield for $L / M$ if and only if $S / K$ is algebraic for some $S$, where $S$ is a $S$-subfield for $L / M$.

Proof. Assume $S / K$ is algebraic for some $S$. Suppose there exists another subfield $S^{\prime}$ of $M$ which is a $S$-subfield for $L / M$. The field $S^{\prime}$ must be algebraically closed in $M$. If $\alpha \in S, \alpha$ is algebraic over $K$, hence $\alpha$ is algebraic over $S^{\prime}$. Since $S^{\prime}$ is algebraically closed in $M, \alpha \in S^{\prime}$. Hence, $S \subseteq S^{\prime}$. But $S$ is maximal, so we conclude $S=S^{\prime}$ and $S$ is unique.

Conversely, suppose $S / K$ is transcendental. Then there exists a finite transcendency basis. Let $\left\{t_{1}, t_{2}, \ldots, t_{r}\right\}$ be such a basis. These elements form a relative $p$-basis for $S / K$; hence they are relatively $p$-independent in $L / K$ by the separability of $L / S\left[5\right.$, p. 378]. Let $M=S\left(\omega_{1}, \omega_{2}, \ldots, \omega_{s}, \beta\right)$ where $\left\{\omega_{1}, \omega_{2}, \ldots, \omega_{s}\right\}$ is a separating transcendency basis for $M / S$ and $\beta$ is separable algebraic over $S\left(\omega_{1}, \omega_{2}, \ldots, \omega_{s}\right)$. There is at least one $\omega_{i}$ since $M / S$ cannot be algebraic. Consider the subfield $K\left(\omega_{1}+t_{1}, t_{2}, \ldots, t_{r}\right)$, a pure transcendental extension of $K$. If $\left(\omega_{1}+t_{1}\right) \in K\left(L^{p}\right)\left(t_{2}, t_{3}, \ldots, t_{r}\right)$ and $\omega_{1} \in K\left(L^{p}\right)\left(t_{2}, t_{3}, \ldots, t_{r}\right)$, then $t_{1} \in K\left(L^{p}\right)\left(t_{2}, t_{3}, \ldots, t_{r}\right)$. This is impossible since $\left(t_{1}, t_{2}, \ldots, t_{r}\right)$ is relatively $p$-independent in $L / S$. Hence, either $\omega_{1}+t_{1}$ or $\omega_{1}$ does not belong to $K\left(L^{p}\right)\left(t_{2}, t_{3}, \ldots, t_{r}\right)$. Let $t_{1}^{\prime}$ represent the element $\omega_{1}$ or $\omega_{1}+t_{1}$ not contained in $K\left(L^{p}\right)\left(t_{1}, t_{2}, \ldots, t_{r}\right)$. Let $S^{\prime}=K\left(t_{1}^{\prime}, t_{2}, \ldots, t_{r}\right)$ and let $\bar{S}^{\prime}$ be its algebraic closure in $M$. The set $\left\{t_{1}^{\prime}, t_{2}, \ldots, t_{r}\right\}$ is relatively $p$-independent in $L / K$ and $\left\{t_{1}^{\prime}, t_{2}, \ldots, t_{r}\right\}$ is a transcendency basis for $\bar{S}^{\prime} / K$, hence a relative $p$-basis for $\bar{S}^{\prime} / K$. By [5, Lemma 3, p. 382], $L / \bar{S}^{\prime}$ is separable. Since $r=\operatorname{tr} \mathrm{d} M / \bar{S}^{\prime}=\operatorname{insep} L / M, \bar{S}^{\prime}$ is a $S$-subfield for $L / M$ [Theorem 8]. By the construction of $S^{\prime}, t_{1}^{\prime} \notin S$; hence, $\bar{S}^{\prime} \neq S$ and $S$ is not unique. 
ACKNOWLEDGEMENT. Most of the results were contained in my thesis under the direction of Dr. James K. Deveney to whom I express my gratitude.

\section{BIBLIOGRAPHY}

1. Jean Dieudonné, Sur les extensions transcedants separables, Summa Brasil Math. 2 (1947), 1-20.

2. Nathan Jacobson, Lectures in abstract algebra. III, Van Nostrand, Princeton, N.J., 1964.

3. H. Kraft, Inseparable Korpenweiterrungen, Comment. Math. Helv. 45 (1970), 110-118.

4. Serge Lang, Algebra, Addison-Wesley, Reading, Mass., 1965.

5. Saunders Mac Lane, Modular fields. I: Separating transcendency bases, Duke Math. 5 (1939), 372-396.

Department of Mathematical Sciences, Virginia Commonwealth University, Richmond, VIRGINIA 23284 